\title{
A Note on Finding all Homogeneous Set Sandwiches
}

\author{
Michel Habib* $\quad$ Emmanuelle Lebhar ${ }^{\dagger} \quad$ Christophe Paul $^{* \ddagger}$
}

26th November 2002

\begin{abstract}
A homogeneous set is a set of vertices $H$ of a graph $G=(V, E)$ such that each vertex of $V \backslash H$ is either adjacent to all vertices of $H$ or to none of them. A graph $G_{s}=\left(V, E_{s}\right)$ is a sandwich for a pair of graph $G_{t}=\left(V, E_{t}\right)$ and $G=(V, E)$ if $E_{t} \subseteq E_{s} \subseteq E$. In a recent paper of Tang et al [4], a $\mathcal{O}\left(\Delta n^{2}\right)$ algorithm is described for computing all the possible homogeneous sets for the sandwich graphs of $G_{t}=\left(V, E_{t}\right)$ and $G=(V, E)$. In this paper, we invalidate this algorithm by proving there are possibly exponentially many such sets. We then give a correct characterization of a homogeneous set of a sandwich graph.
\end{abstract}

Keywords : analysis of algorithms, graphs, graph sandwich problems, homogeneous set.

\section{Introduction}

A graph $G_{t}=\left(V, E_{t}\right)$ is a spanning subgraph of $G=(V, E)$ if $E_{t} \subseteq E$. A graph $G_{s}=\left(V, E_{s}\right)$ is a sandwich graph for the pair $G$ and $G_{t}$ if $E_{t} \subseteq E_{s} \subseteq E$. The GRAPH SANDWICH PROBLEM for a property $\Pi$ is defined as follows:

Problem 1: Graph SANDWich PROBlem For Property $\Pi$

Instance: $\quad$ Two graphs $G_{t}=\left(V, E_{t}\right)$ and $G=(V, E)$ such that $E_{t} \subseteq E$

Question: Does there exist a sandwich graph $G_{s}=\left(V, E_{s}\right)$ for the pair $\left(G, G_{t}\right)$ satisfying property $\Pi$ ?

Notice that a sandwich problem for property $\Pi$ can be seen has a generalization of the recognition problem for property П. Graph sandwich problems has been introduced by Golumbic, Shamir and Kaplan [2]. In their seminal paper, they study a wide range of sandwich problems for subfamilies of perfect graphs. Unfortunately many of these sandwich problems appear to be NP-complete. Strangely the homogeneous sandwich problem is polynomial and recently two papers [1] and [4] present efficient algorithms to solve it.

\section{Problem 2: Homogeneous Set SANDWich PROBlem}

Instance: $\quad$ Two graphs $G_{t}=\left(V, E_{t}\right)$ and $G=(V, E)$ such that $E_{t} \subseteq E$

Question: Does there exist a sandwich graph $G_{s}=\left(V, E_{s}\right)$ for the pair $\left(G, G_{t}\right)$ containing a homogeneous set?

A homogeneous set (or a module) is a set of vertices $H$ of a graph $G=(V, E)$ such that each vertex of $V \backslash H$ is either adjacent to all vertices of $H$ or to none of them [3]. The first polynomial algorithm to this problem is due to Cerioli et al. [1]. This algorithm has $\mathcal{O}\left(n^{4}\right)$ time-complexity, where $n$ represents the number of vertices in the instance and finds a homogeneous set sandwich

\footnotetext{
*LIRMM-Université de Montpellier II, 161 rue Ada, 34392 Montpellier Cedex, France

†Laboratoire de l'Informatique du Parallélisme, École Normale Supérieure de Lyon, 46 Allée d'Italie, 69364 Lyon Cedex 07, France

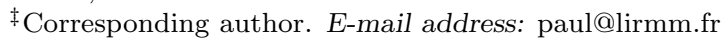


if there exists one. By using, what they called, the bias graph, Tang et al [4] claimed that the number of homogeneous set sandwiches is bounded by $n^{2}$ and provided a better time complexity with an $\mathcal{O}\left(\Delta n^{2}\right)$ algorithm, where $\Delta$ is the maximum degree in $G$. In this paper, we invalidate these statements by proving there are possibly exponentially many homogeneous set sandwiches.

Hereafter we deal with instance of the homogeneous set sandwich problem for graphs $G_{t}=$ $\left(V, E_{t}\right)$ and $G=(V, E)$. Section 2 summarizes Tang et al.'s paper. In section 3, we propose a precise definition of the homogeneous set sandwich enumerative problem and show that the number of homogeneous set sandwiches can be exponential in the size of the instance. Section 4 gives a characterization of homogeneous set sandwiches, which corrects Tang et al.'s result.

\section{$2 \quad$ Tang et al's results}

A bias vertex $u$ of a vertex set $S \subseteq V \backslash\{u\}$ is a vertex such that there exist two vertices $v, w \in S$ verifying $u v \in E_{t}$ and $u w \notin E$. A bias set $C$ of vertex set $S$ for graphs $G$ and $G_{t}$ is a set containing all bias vertices of $S$. We give here the proof of the following characterization on which Tang et al.'s algorithm is based, which was somehow implicit in this article.

Theorem 2.1 $A$ vertex set $S \subseteq V$ is a homogeneous set for some sandwich graph if and only if the bias set of $S$ is empty.

Proof: If there exists vertex $s \in V \backslash S$ which is a bias vertex for $S$, then there are two vertices in $S$ which have distinct neighborhoods, thus $S$ is not a homogeneous set for any sandwich graph.

For the converse if $S$ is not a homogeneous set in any graph sandwich, there are two vertices $s_{1}, s_{2} \in S$ having distinct neighborhoods for $E$ in $V \backslash S$. Then there exists a vertex $x \in V \backslash S$ such that $x s_{1} \in E_{t}$ and $x s_{2} \notin E$, thus $s$ is a bias vertex for $S$, its bias set is not empty.

The bias graph $G_{b}=\left(V_{b}, E_{b}\right)$ of a pair of graphs $G_{t}=\left(V, E_{t}\right), G=(V, E)$ has vertex set $V \times V$ and there are two outgoing edges from vertex $(u, v)$ to vertices $(u, w)$ and $(v, w)$ in $G_{b}$ if and only if vertex $w$ is a bias vertex of vertex set $\{u, v\}$. From this structure follows the decision algorithm based on Theorem 2.2:

Theorem 2.2 ([4]) Any strongly connected component in the bias graph is a homogeneous set sandwich.

The condensation graph $G_{c}=\left(V_{c}, E_{c}\right)$ of a digraph $G_{b}=\left(V_{b}, E_{b}\right)$ has each of its vertices corresponding to a strongly connected component in $G_{b}$ and labeled as the union of all vertices in the component. All outgoing edges of a strongly connected component in $G_{b}$ are replaced by one outgoing edge of the corresponding vertex in $G_{c}$. In the condensation graph of a digraph $G_{b}$, the union of a vertex $v$ and all the vertices $u$ such that there exists a path from $v$ to $u$ is a vertex set called a $r$-union set ( $r$ standing for reachable union set [4])).

Claim $2.1([4]) H$ is a homogeneous set for some sandwich graph if and only if $H$ is a $r$-union set and $H \neq V$.

Since the condense graph has at most $n^{2}$ nodes, from the above claim one can conclude there are at most $n^{2}$ homogeneous set sandwiches. Indeed the authors announced an $\mathcal{O}\left(\Delta n^{2}\right)$ algorithm which should find all homogeneous set sandwiches. As we will see in the next sections, it is not possible since we are able to show an instance of graph sandwich with at least $4^{n}$ homogeneous set sandwiches.

\section{$3 \quad$ Finding all homogeneous set sandwiches}

In some applications for example when vertices are equipped with weights, a natural problem is to find a minimum weighted homogeneous set sandwich. A first step in this direction is to enumerate all homogeneous set sandwiches. First we must detail which homogeneous sets are relevant in 
the enumerative problem. Indeed, trying to find all homogeneous sets without restriction leads to exponential enumeration given that the number of all homogeneous sets in a graph is often exponential in the size of the graph (as for example, when a graph is complete or empty, every subset of vertices is a homogeneous set).

To avoid these artefacts, the following notion of the essential or strong homogeneous sets has been successfully introduced. A homogeneous set $M$ of a graph $G=(V, E)$ is a strong homogeneous set if and only if for any homogeneous set $M^{\prime}$ such that $M^{\prime} \cap M \neq \emptyset$, either $M \subseteq M^{\prime}$ or $M^{\prime} \subseteq M$. Strong homogeneous sets of a graph $G$ are homogeneous sets that appear in the modular decomposition tree of $G$, by the way there are $\mathcal{O}(n)$ strong homogeneous sets. Consequently in the enumerative problem, we are interested in finding strong homogeneous sets only because the other ones will be insignificant for the decomposition tree.

\section{Problem 3: Homogeneous set SAndwich enumerative Problem}

Instance: $\quad$ Two graphs $G_{t}=\left(V, E_{t}\right)$ and $G=(V, E)$ such that $E_{t} \subseteq E$

Question: Find every pairs $\left\{H, G_{s}=\left(V, E_{s}\right)\right\}$ where $H$ is a strong homogeneous set, and $G_{s}$ the corresponding sandwich graph such that $E_{s}$ is minimal for the inclusion.

Even if we restrict our attention to strong homogeneous sets, the following proposition prevents from any polynomial enumeration for the above problem.

Theorem 3.1 The number of strong homogeneous set sandwiches can be exponential in the size of the instance.

Proof: Let us consider the example described by figure 1 . The only bias vertices are the $x_{i}$ 's with $1 \leq i \leq n$ : they are bias vertices for $\left\{y_{i}, z_{i}\right\}$.

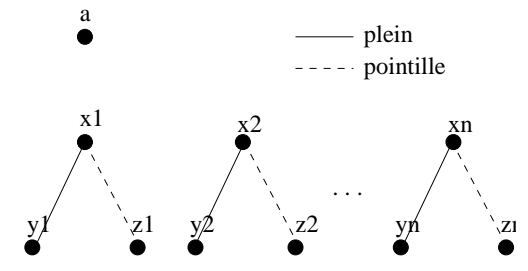

Figure 1: Instance having an exponential number of strong homogeneous set sandwiches.

Let us now enumerate all strong homogeneous sets containing vertex $a$. Let us consider one triplet $\left\{x_{i}, y_{i}, z_{i}\right\}$, we can find 3 strong homogeneous sets that contains $a: M_{i, 1}=\left\{a, x_{i}\right\}$ which is strong in sandwich graph $G_{i, 1}^{s}=\left(V, E_{i, 1}^{s}\right)$ where $E_{i, 1}^{s}=E_{t} \cup\left\{a y_{i}\right\}$ is the minimal set of edge for the inclusion, similarly $M_{i, 2}=\left\{a, y_{i}\right\}$ and $M_{i, 3}=\left\{a, x_{i}, z_{i}\right\}$ are strong too in their sandwich graph. Strong homogeneous sets corresponding to each triplet can be gathered arbitrarily to give new strong homogeneous sets thanks to the following statement.

Statement 3.1 Let $M_{i, 1}, M_{i, 2}, M_{i, 3}$ be the three strong homogeneous set sandwiches associated to triplet $\left\{x_{i}, y_{i}, z_{i}\right\}$ and $M_{i, 0}$ be the empty set associated to this triplet.

$$
M=\bigcup_{i=1}^{n} M_{i, k_{i}}, \text { with } k_{i} \in\{0,1,2,3\} \forall i \leq n
$$

The set $M$ is a strong homogeneous set for sandwich graph $G_{s}=\left(V, E_{s}\right)$ where $E_{s}=E_{t} \cup\{u v \mid u \in$ $M, v \in V \backslash M, \exists w \in M$ with $\left.w v \in E_{t}\right\}$.

Statement proof: If $M=\emptyset$ the proof is trivial. Suppose $M \neq \emptyset$. By definition of $E_{s}$, vertices of $M$ have the same neighborhood outside $M$. Indeed suppose that vertex $u \in M$ is linked to vertex $v \notin M$ by edge $e \in E_{s}$. If $e \in E_{t}$ then $\forall x \in M, x v \in E_{s}$ by definition of $E_{s}$. If $e \in E_{s} \backslash E_{t}$ 
there is a vertex $w \in M$ such that $w v \in E_{t}$ and the same argument shows that $\forall x \in M, x v \in E_{s}$, finally $M$ is a homogeneous set in $G_{s}$.

And $M$ is a homogeneous set sandwich because $E_{s} \subseteq E$. Indeed the only edges not in $E$ are edges $\left\{x_{i} z_{i}\right\}_{1 \leq i \leq n}$, suppose that there exists $i_{0}$ such that $x_{i_{0}} z_{i_{0}} \in E_{s}$. Since $z_{i_{0}} \in M$ implies $x_{i_{0}} \in M$ (from the three possible homogeneous set sandwiches of the triplet), $x_{i_{0}} \in M$ and $z_{i_{0}} \notin M$. As $x_{i_{0}} z_{i_{0}} \in E_{s}$, there exists $w \in M$ such that $z_{i_{0}} w \in E_{t}$, this is a contradiction because no edge of $E_{t}$ is adjacent to any vertex of $\left\{z_{i}\right\}_{1 \leq i \leq n}$ in the instance, thus $E_{s} \subseteq E$.

Suppose by contradiction that $M$ is not strong in $G_{s}$, then there exists a homogeneous set $M^{\prime}$ such that: $M \cap M^{\prime} \neq \emptyset, \exists u^{\prime} \in M^{\prime} \backslash M$.

As each homogeneous set $M_{i, k_{i}}$ implies to add an edge of $E \backslash E_{t}$, because of vertex $a$, to build a sandwich graph appropriate to it (if $k_{i} \neq 0$ ), then the number of edges in $G_{s}$ is strictly greater than the number of edges in $G_{t}$ since $M \neq \emptyset$.

As $M \neq \emptyset$ there exists $i_{0} \leq n$ such that $k_{i_{0}} \neq 0$, homogeneous set $M_{i_{0}, k_{i_{0}}}$ is included in $M$ and so either $x_{i_{0}}$ or $y_{i_{0}}$ belongs to $M$ but not both. Finally since $x_{i_{0}} y_{i_{0}} \in E_{t}$, there exists $u v \in E_{t}$ with $u \in M, v \notin M$. As $M \cap M^{\prime} \neq \emptyset, M$ and $M^{\prime}$ have the same neighbors and there exists an edge $e^{\prime}=\left\{u^{\prime}, v\right\}$ in $G_{s}$.

As there is no path of length 2 in $G_{t}, e^{\prime}$ is not in $E_{t}$. As the edge $u^{\prime} v$ has no end in $M, e$ does not belong to $E_{s} \backslash E_{t}=\left\{u v \in E \mid u \in M, v \notin M, \exists w \in M\right.$ with $\left.w v \in E_{t}\right\}$. Finally $e^{\prime}$ does not belong to $G_{s}$ and $M^{\prime}$ is not a homogeneous set of $G_{s}$, this is a contradiction thus $M$ is a strong homogeneous set sandwich for $G_{s}$. This ends statement proof.

To complete the proof of theorem, one can notice that sandwich graph $G_{s}$ of statement 3.1 is the sandwich graph associated to $M$ that has an edge set minimal for the inclusion. As we can build distinct sets $M$ with every combinations of the elements of $\left\{M_{i, k_{i}}\right\}_{1 \leq i \leq n}$, since $\forall i \in$ $[1, n], k_{i} \in\{0,1,2,3\}$, there are $4^{n}$ strong sandwich homogeneous sets in this example.

\section{Characterization of homogeneous set sandwiches}

Tang et al erroneously proposed a characterization of homogeneous set sandwiches as the r-union sets (see statement 2.1). It is clear from theorem 2.2 that any r-union set is a homogeneous set for a sandwich graph. Unfortunately, as shown by the example of figure 2 (its bias graph is described in figure 3), the converse is not true.

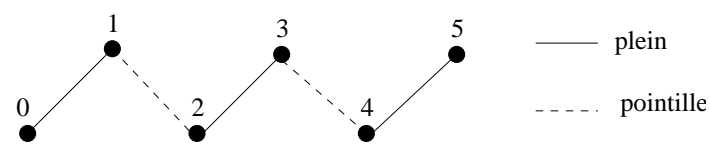

Figure 2: Homogeneous set sandwich instance $I_{e x}$.

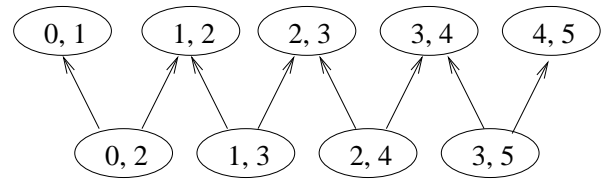

Figure 3: Condensation of bias graph corresponding to $I_{e x}$.

One can read all r-union sets on figure $3:\{0,1\},\{1,2\},\{2,3\},\{3,4\},\{4,5\},\{0,1,2\},\{1,2,3\}$, $\{2,3,4\},\{3,4,5\}$. But, for instance, the set $S=\{0,1,2,3,4\}$ is also a homogeneous set for the sandwich graph:

$$
G_{s}=(V,\{\{0,1\},\{2,3\},\{4,5\},\{3,5\},\{0,5\},\{1,5\},\{2,5\}\})
$$


$S$ is a strong homogeneous set in $G_{s}$ but does not appear as a r-union set. Therefore claim 2.1 is mistaken. Since Tang et al's algorithm enumerates all r-union sets, it misses the set $S$.

Let us now propose a correct characterization of homogeneous set sandwiches using the operation $\sigma$ defined below.

Definition 4.1 Let $S$ be a subset of $V$. Operation $\sigma$ is defined on $V$ by:

$$
\sigma(S) \stackrel{\text { def }}{=} \bigcup_{a, b \in S} r \text {-union }\left(N_{a, b}\right)
$$

Where $N_{a, b}$ is the unique node in $G_{c}$ corresponding to the strongly connected component of $G_{b}$ that contains node $(a, b)$.

Cerioli et al. gave in [1] an algorithm to decide whether there exists a homogeneous set for some sandwich graph by computing the set of bias vertices $H_{a, b}^{1}$ of two given vertices $a, b \in V$, then the set $H_{a, b}^{2}=H_{a, b}^{1} \cup\left\{\right.$ bias vertices of $\left.H_{a, b}^{1}\right\}$ onto a set $H_{a, b}^{s}$ having an empty bias set. This operation is polynomial in $\mathcal{O}\left(n^{2}\right)$. Operation $\sigma$ does the same sets except that it starts from an arbitrary set $S$ and not just from a couple of vertices. Its closure can be computed is $\mathcal{O}\left(\Delta n^{2}\right)$ by Tang et al's algorithm since in worst case it has to find all r-union sets. As shown by the following theorem, any homogeneous set sandwich is obtained as the closure of some set $S$ by the operation $\sigma$. Therefore an enumeration algorithm should do this computation for any set $S$ of vertices which implies that time complexity grows to a number exponential in the size of the instance.

Theorem 4.1 (Characterization of homogeneous set sandwiches) $H$ is a homogeneous set if and only if $H \neq V$ and $H=\sigma(H)$

Proof: Let $H \subset V$ be a homogeneous set sandwich, we show that $H=\sigma(H)$. Suppose by contradiction there exits $x \notin H$ such that $x \in \mathrm{r}$-union $\left(N_{a, b}\right)$ for $a, b \in H$, then whether $x \in N_{a, b}$, or $x \in \mathrm{r}$-union $\left(N_{a, b}\right) \backslash N_{a, b}$. If $x \in N_{a, b}$ it means that $x$ is in the same strongly connected component as node $(a, b)$ in $G_{b}$. Then there is a path in $G_{c}$ from $(a, b)$ to a node containing $x$, and $x$ is a bias vertex for $\{a, b\}$. This is a contradiction because if $H$ is a homogeneous set sandwich, its bias set is empty by theorem 2.1. Similarly, if $x \in \mathrm{r}$-union $\left(N_{a, b}\right) \backslash N_{a, b}$ for $a, b \in H$, it would be a bias vertex for $H$ which is a contradiction, so $\forall a, b \in H$, r-union $\left(N_{a, b}\right) \subseteq H$ and finally $\sigma(H)=\bigcup_{a, b \in H}$ r-union $\left(N_{a, b}\right) \subseteq H$. As every vertices of $H$ are in $\sigma(H)$ by definition of operation $\sigma$, the equality $H=\sigma(H)$ follows.

For the converse, let $S$ be a subset of $V$ such that $S=\sigma(S)$ and $S \neq V$, we show that $S$ is a homogeneous set sandwich. Suppose by contradiction that $x \in V \backslash S$ is a bias vertex for vertices $a, b \in S$, then node $(a, b)$ in $G_{b}$ has two outgoing edges to nodes $(a, x)$ and $(b, x)$. So $x \in \mathrm{r}$-union $\left(N_{a, b}\right) \subseteq \sigma(S)$, as $\sigma(S)=S$, we have $x \in S$ and $x$ cannot be a bias vertex for $S$ which is a contradiction. Finally, $S$ is a homogeneous set sandwich.

\section{Concluding remarks}

This paper shows that the enumerative homogeneous set sandwich problem is not polynomial whereas the corresponding decision problem is. Deciding whether this enumerative problem is \#P-complete is an open question.

The exponential number of strong homogeneous set sandwiches gives credit for the NP-hardness of the weighted homogeneous set sandwich problem, which deals with determining a homogeneous set in a weighted sandwich graph having a minimal weight. Indeed, if it is necessary to compute the weight of every homogeneous sets to get the minimal one, this computation can be exponential from 3.1 .

The homogeneous set edge-completion problem that asks for the minimal number of edges to add to form a homogeneous set in $G$ which also needs to compute all homogeneous set sandwiches between two graphs $\left(G\right.$ and $\left.K_{n}\right)$ [4] should neither be solved polynomially. More generally, it should be very interesting to investigate the following problem for various property $\Pi$ : 
Problem 4: Minimum edge completion sandwich problem for property $\Pi$

Instance: Two graphs $G_{t}=\left(V, E_{t}\right)$ and $G=(V, E)$ such that $E_{t} \subseteq E$ and an integer $k$

Question: Does there exist a sandwich graph $G_{s}=\left(V, E_{s}\right)$ with property $\Pi$ such that $\left|E_{s}\right|$ $E_{t} \mid \leqslant k ?$

\section{References}

[1] M. R. Cerioli, H. Everett, C. M. H. de Figueiredo, and S. Klein. The homogeneous set sandwich problem. Information Processing Letters, 67:31-35, 1998.

[2] M. C. Golumbic, H. Kaplan, and R. Shamir. Graph sandwich problems. Journal of Algorithms, 19:449-473, 1995.

[3] L. Lovász. Normal hypergraphs and the perfect graph conjecture. Discrete Mathematics, $2: 253-267,1972$.

[4] S.-M. Tang, F.-L. Yeh, and Y.-L. Wang. An efficient algorithm for solving the homogeneous set sandwich problem. Information Processing Letters, 77:17-22, 2001. 